\title{
Holographic recording in a photopolymer by optically induced detachment of chromophores
}

\author{
Gregory J. Steckman \\ Department of Electrical Engineering, California Institute of Technology, Mail Stop 136-93, Pasadena, California 91125 \\ Vladimir Shelkovnikov, Victoria Berezhnaya, and Tatiana Gerasimova \\ Novosibirsk Institute of Organic Chemistry, 9 Academic Lavrentjev Avenue, Novosibirsk 630090, Russia \\ Iouri Solomatine and Demetri Psaltis \\ Department of Electrical Engineering, California Institute of Technology, Mail Stop 136-93, Pasadena, California 91125
}

Received January 7, 2000

\begin{abstract}
We demonstrate holographic recording in a new photopolymer system. The recording material is created by copolymerization of an optically inert monomer, methyl methacrylate, and a second monomer that is optically sensitive. On exposure of the recording material to light, a portion of the optically sensitive component detaches from the polymer matrix and causes hologram amplification through diffusion of the free molecules. We measured postrecording grating amplifications as high as $170 \%$ by this process. The recorded holograms are persistent at room temperature under continuous illumination at the recording wavelength. (c) 2000 Optical Society of America
\end{abstract}

OCIS codes: $\quad 090.2900,160.5470,210.4810,350.5130$.

Photopolymer materials are promising candidates for holographic data storage. ${ }^{1-3}$ One such photopolymer that has been investigated in the past is phenanthrenequinone-doped poly(methyl methacrylate). ${ }^{4-7}$ In this system, when the dopant chromophore phenanthrenequinone $(\mathrm{PQ})$ is illuminated it undergoes a chemical reaction that causes it to attach to the host poly(methyl methacrylate) (PMMA) matrix. When the material is illuminated with an interference pattern, two gratings are formed. The first consists of $\mathrm{PQ}$ molecules that are attached to the polymer matrix, and the second consists of unattached PQ molecules. Subsequent baking of the material causes an acceleration in the diffusion rate of the unattached $\mathrm{PQ}$ molecules, which causes an overall amplification of the resulting diffraction efficiency. In this new material system, chromophores are initially attached to the polymer chains. This attachment is accomplished by synthesis of a monomer containing a photosensitive side chain that exhibits the desired photochemical properties and copolymerizing it with a monomer that forms a known high-optical-quality material, such as PMMA. On illumination of the material, the attached chromophores undergo a photochemical reaction, causing them to detach from the polymer backbone. When the material is illuminated with an interference pattern, a grating composed of two components, attached and detached chromophores, results, as in the case of the PQ-doped system. Heating of the material causes an amplification of the grating strength through diffusion of the detached molecules.

Figure 1 shows the material that we synthesized and the process of detaching the chromophore from a polymer chain. The optically active component, shown in Fig. 1(a), is the synthesized monomer $N, N^{\prime}$-dimethyl- $N$-(3-methyl-1, 4-naphthoquinonyl-2)$N^{\prime}$-acryloyl-hexamethylenediamine. This monomer is based on the chromophore 1,4-naphthoquinone (NQ) [left-hand side of Fig. 1(a)], attached by an alkyl chain (center) to the right-hand section, which can take part in copolymerization with methyl methacrylate. Copolymerizing the synthesized monomers with the monomer methyl methacrylate forms a mostly PMMAbased material. The copolymer structure is shown in Fig. 1(b), illustrating the process occurring when the chromophore is present as a side chain in the polymer.

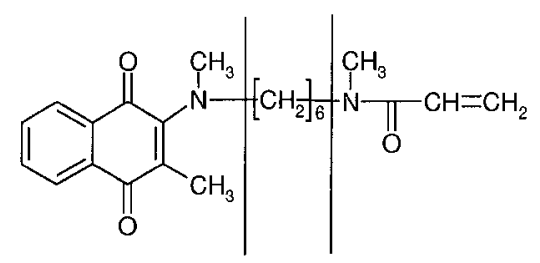

(a)

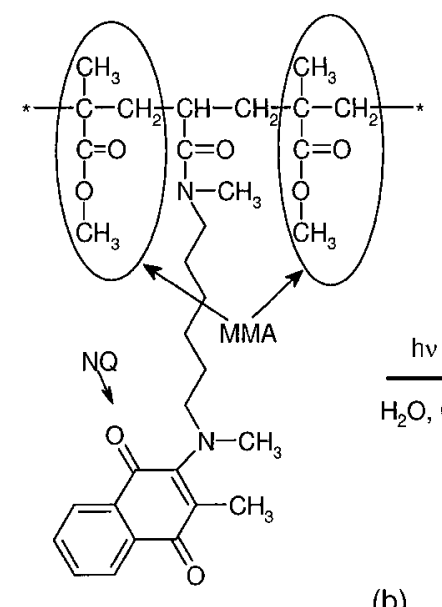

(b)

Fig. 1. (a) Synthesized monomer form of the NQ chromophore. (b) Copolymer of the NQ chromophore with methyl methacrylate and the photochemical reaction that causes detachment from the polymer chain. 
When it is illuminated, $N$-naphthoquinonylamine undergoes a transformation first to a metastable state before it is transformed into its final stable form. ${ }^{8}$ A portion of the chromophore detaches from the polymer and is free to diffuse as discussed above.

A basic holographic recording system was used to test the material. The system consisted of two 514-nm recording beams, each incident at a $20^{\circ}$ angle (in air) from the material's surface normal. A Braggmatched $633-\mathrm{nm}$ probe beam was used to read out the holograms. The intensity of each recording beam was $20 \mathrm{~mW} / \mathrm{cm}^{2}$. The absorption spectrum of this material, with a chromophore concentration of $0.1 \%$ by weight and thickness of $0.5 \mathrm{~mm}$ (see Fig. 2), shows little to no absorption at the 633-nm probe wavelength and significant absorption at 514-nm, owing to the added chromophore.

Samples were prepared with chromophore concentrations of $0.05 \%, 0.1 \%, 0.2 \%$, and $0.4 \%$ by weight. Eleven holograms were recorded in each sample, at different locations, with exposure energies varying from 0.04 to $1.6 \mathrm{~J} / \mathrm{cm}^{2}$. Each recorded hologram was then measured after the sample was baked for 11 days at $75^{\circ} \mathrm{C}$. Figure 3 contains a plot of the measured diffraction efficiencies before and after baking of a $0.1 \%$ by weight, $0.6-\mathrm{mm}$-thick sample. As expected, the recorded holograms were amplified after baking, supporting the assumption of hologram recording and amplification through the optical detachment of chromophore side chains and subsequent diffusion. The eventual decrease in hologram strength with increasing exposure energy is an expected behavior of saturable recording materials, which do not exhibit significant diffusion at room temperature. ${ }^{6}$

So far, materials have been prepared with a chromophore concentration as high as $0.4 \%$ by weight. The total index modulation to be expected should increase roughly linearly with increased chromophore concentration. In PQ-doped PMMA the dynamic range is limited by the maximum doping level of the $\mathrm{PQ}$ chromophores. A maximum concentration of $0.7 \%$ by weight has been achieved, ${ }^{6}$ resulting in $M / \#=4.8$ for a $3-\mathrm{mm}$-thick sample. The concentration is limited by the solubility of $\mathrm{PQ}$. The approach that we present in this Letter can in principle support a higher concentration of the active chromophore. However, for higher-concentration material we must switch to a longer recording wavelength and redesign the attached chromophore so that the absorption is reduced without sacrificing sensitivity. With current absorption levels, at $514 \mathrm{~nm}$, even $0.4 \%$ concentrations are too high, which limits our ability to record thick holograms. As a result, the overall diffraction efficiencies are reduced and the angular selectivity is broadened. Figure 4 is a comparison of the angle-selectivity curves, measured at $633 \mathrm{~nm}$, obtained for a hologram recorded at $514 \mathrm{~nm}$ in a sample with a chromophore concentration of $0.05 \%$ and a sample with a chromophore concentration of $0.4 \%$, with the same thickness. We normalized and centered the selectivity curves to facilitate comparison. It is easy to see well-defined nulls for the $0.05 \%$ sample. However, the selectivity curve for the $0.4 \%$ sample does not have nulls and is also broader, indicating that the hologram is not recorded throughout the volume of the material but rather varies in strength throughout the thickness of the material.

The grating strength of the hologram recorded with optimal exposure energy is plotted in Fig. 5 for samples with four different $\mathrm{NQ}$ concentrations. A linear increase in grating strength with concentration

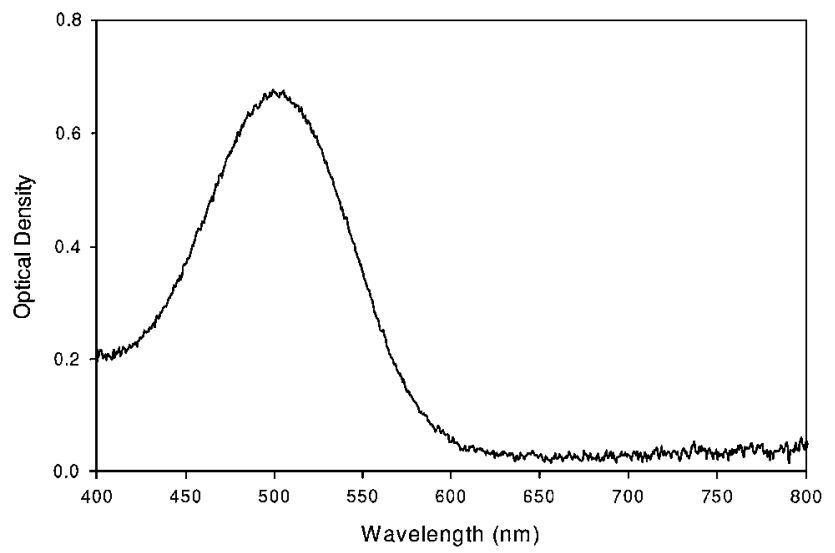

Fig. 2. Absorption spectrum for a 0.5 -mm-thick sample with a chromophore concentration of $0.1 \%$ by weight.

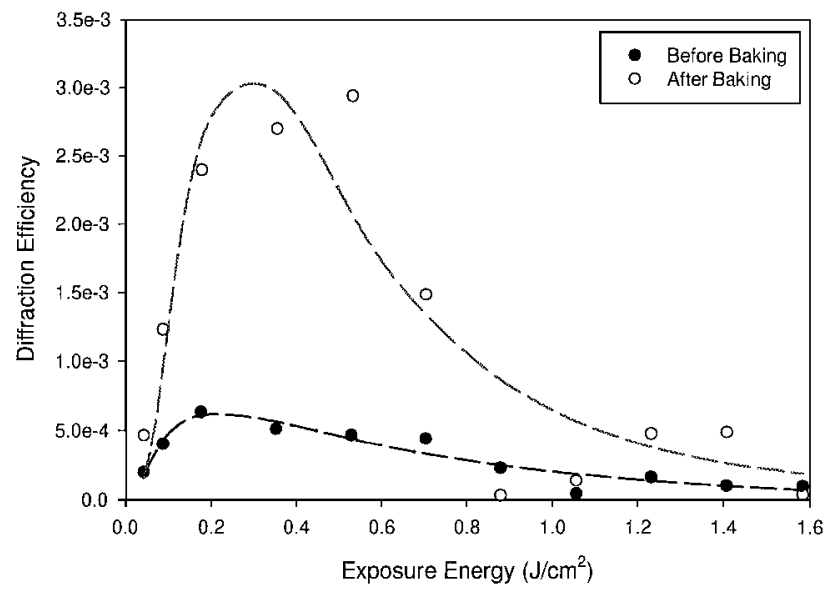

Fig. 3. Diffraction efficiency versus exposure energy for 11 holograms recorded in a $0.6-\mathrm{mm}$-thick sample with a chromophore concentration of $0.1 \%$ by weight, before and after baking.

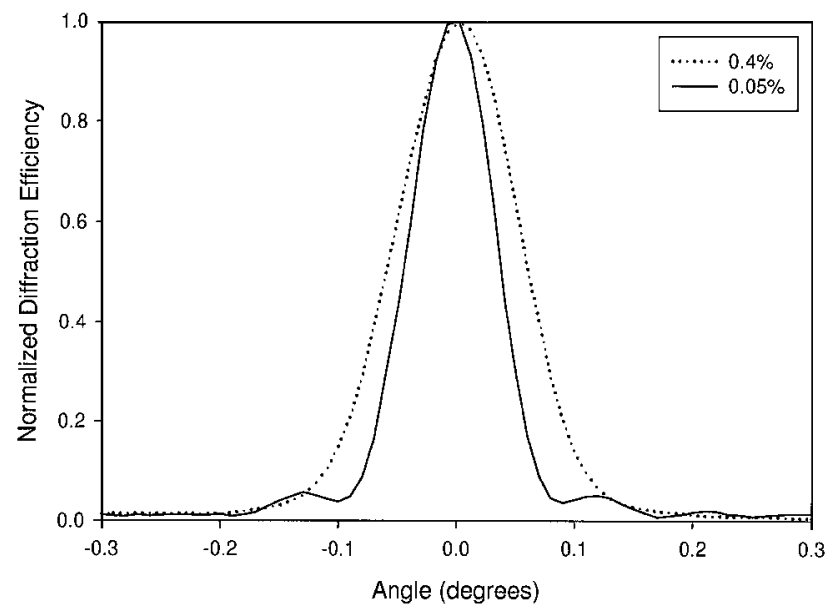

Fig. 4. Angle-selectivity measurements of holograms recorded in materials with $0.05 \%$ and $0.4 \%$ chromophore concentrations. 


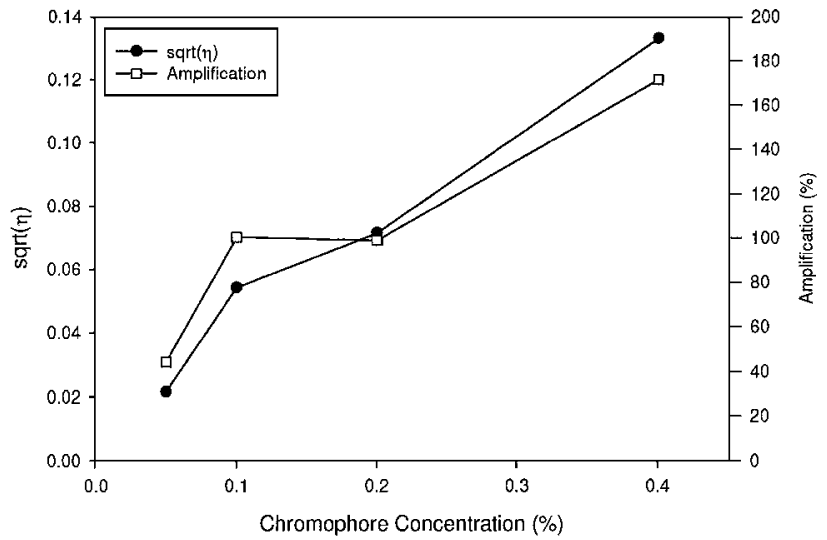

Fig. 5. Dependence of the maximum diffraction efficiency and amplification of the recorded holograms in the NQ methyl methacrylate copolymer, after 11 days of baking, on the chromophore concentration. The samples were $0.5 \mathrm{~mm}$ thick.

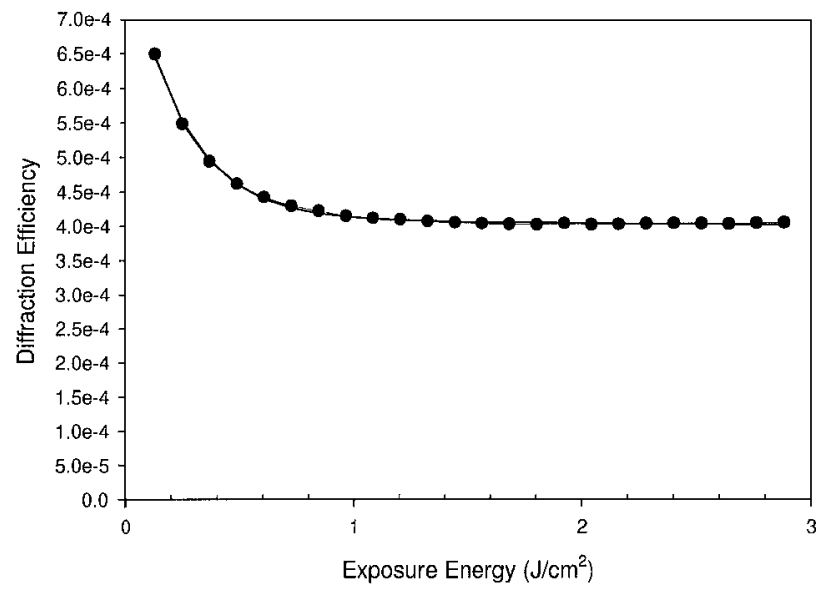

Fig. 6. Decay in hologram strength with exposure to illumination after recording and thermal amplification owing to the detachment of the remaining attached chromophores.

is observed. Also plotted in Fig. 5 is the average amplification of the grating strengths of the 11 holograms in each sample after 11 days of baking at $75^{\circ} \mathrm{C}$ as a function of chromophore concentration. Amplifications of $50-170 \%$ were realized. One possible explanation for the increase with concentration is that for higher concentrations the grating is not uniform throughout the volume of the material, resulting in detached chromophore diffusion in directions normal to the grating vector rather than strictly parallel to it, as would be the case with a uniform modulation depth.

Figure 6 shows the diffraction efficiency as a function of exposure energy when a recorded hologram is exposed to uniform illumination at $514 \mathrm{~nm}$. There is an initial drop in diffraction efficiency of approximately $40 \%$ at $\sim 0.5 \mathrm{~J} / \mathrm{cm}^{2}$. Subsequently, the diffraction efficiency remains unchanged, yielding nondestructive readout. The initial decay is due to the detachment of the remaining chromophores, which causes an index change similar to the effect that results in the observed diffraction efficiency before baking. However, at room temperature the nonuniformity of the detached chromophores remains in place and gives persistent diffraction.

This effort was sponsored by the Defense Advanced Research Projects Agency and the U.S. Air Force Research Laboratory, Air Force Material Command, under agreement F30602-98-1-0199. This work was also supported as part of the Neuromorphic Systems Engineering Research Center. G. J. Steckman acknowledges support from an Intel Foundation fellowship; his e-mail address is steckman@caltech.edu.

\section{References}

1. L. Dhar, K. Curtis, M. Tackitt, M. Schilling, S. Campbell, W. Wilson, and A. Hill, Opt. Lett. 23, 1710 (1998).

2. S. Blaya, L. Carretero, R. Mallavia, A. Fimia, R. F. Madrigal, M. Ulibarrena, and D. Levy, Appl. Opt. 37, 7604 (1998).

3. D. A. Waldman, H.-Y. S. Li, and M. G. Horner, J. Imaging Sci. Technol. 41, 497 (1997).

4. A. V. Veniaminov, V. F. Goncharov, and A. P. Popov, Opt. Spektrosk. 70, 864 (1991).

5. V. I. Sukhanov, J. Opt. Technol. 61, 49 (1994).

6. G. J. Steckman, I. Solomatine, G. Zhou, and D. Psaltis, Opt. Lett. 23, 1310 (1998).

7. K. Y. Hsu, S. H. Lin, W. T. Whang, and W. Z. Chen, Proc. SPIE 3801, 66 (1999).

8. R. P. Shishkina and V. N. Berezhnaya, Russ. Chem. Rev. 63, 139 (1994). 